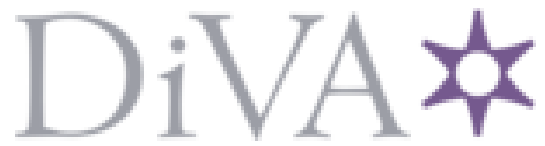

http://www.diva-portal.org

Preprint

This is the submitted version of a paper published in .

Citation for the original published paper (version of record):

Riveiro, M., Pallotta, G., Vespe, M. (2018)

Maritime anomaly detection: A review

Wiley Interdisciplinary Reviews. Data Mining and Knowledge Discovery, 8(5): e1266 https://doi.org/10.1002/widm.1266

Access to the published version may require subscription.

N.B. When citing this work, cite the original published paper.

Permanent link to this version:

http://urn.kb.se/resolve?urn=urn:nbn:se:his:diva-16182 
"This is the pre-peer reviewed version of the following article: "Riveiro M., Pallotta G., and Vespe M. Maritime anomaly detection: A review. WIREs Data Mining Knowl Discov. Advanced Review. 2018;e1266. https:// doi.org/10.1002/widm.1266", which has been published in final form at https://onlinelibrary.wiley.com/doi/ abs/10.1002/widm.1266. This article may be used for non-commercial purposes in accordance with Wiley Terms and Conditions for Use of Self-Archived Versions."

\title{
Maritime Anomaly Detection: A Review
}

\author{
Maria Riveiro ${ }^{1}$, Giuliana Pallotta ${ }^{2}$, and Michele Vespe ${ }^{3}$
}

\begin{abstract}
The surveillance of large sea areas normally requires the analysis of large volumes of heterogeneous, multidimensional and dynamic sensor data, in order to improve vessel traffic safety, maritime security and to protect the environment. Early detection of conflict situations at sea provides critical time to take appropriate action with, possibly before potential problems occur. In order to provide an overview of the state-of-theart of research carried out on the analysis of maritime data for situation awareness, this paper presents a review of maritime anomaly detection. The found articles are categorized into four groups (1) data, (2) methods, (3) systems and (4) user aspects. We present a comprehensive summary of the works found in each category, and finally, we outline possible paths of investigation and challenges for maritime anomaly detection.
\end{abstract}

Keywords: anomaly detection, maritime anomaly detection, maritime traffic, situation awareness, data-mining, review.

\section{INTRODUCTION}

Sea freight carries more than 90\% of the world's global trade (Wan et al. 2016), constituting, thus, the arteries of global transportation. The availability of a larger number of sensors, higher data storage capacity, cheaper devices and better database management systems have made it possible to access huge volumes of data related to the sea traffic. Such volumes of traffic require a form of traffic control, in order to guarantee safety, protect our environment, optimize traffic streams, and avoid illegal activities, such as smuggling.

Usually, surveillance operators have to search and predict emerging conflict situations, e.g., risk for collision, anomalous vessels or suspicious activities from a large number of vessels within vast sea areas. Exploring and monitoring the data

\footnotetext{
${ }^{1}$ School of Informatics, University of Skövde, SE-541 28, Skövde, Sweden E-mail: maria.riveiro@his.se.

${ }^{2}$ Lawrence Livermore National Laboratory, Livermore, CA 94551, USA. E-mail: pallotta2@llnl.gov.

${ }^{3}$ European Commission, Joint Research Centre (JRC), Directorate for Space, Security and Migration, Demography, Migration \& Governance Unit, Via E. Fermi 2749, I-21027 Ispra (VA), Italy. E-mail: michele.vespe@ec.europa.eu.
} 
through surveillance systems may become a demanding activity for operators, not only due to the complexity and heterogeneous nature of the data but also to other factors like uncertainty, fatigue, cognitive overload or other time constraints. Early detection of such situations provides critical time to take appropriate action with, possibly before potential problems occur (Wiersma 2010). In order to provide support for the operators in these situations, methods and systems with anomaly detection capabilities are being introduced and used widely.

Detecting outliers and anomalies in data has been studied in the statistics community as early as the 19th century (Chandola et al. 2009), even if the earliest work on the application of anomaly detection in this area we found is from the 90s. Anomaly detection in the maritime domain was identified by the operators/analysts of the operational community as an important aspect requiring research and development (Martineau and Roy 2011). The area of maritime anomaly detection is an active research area which has been receiving increasing attention from several research communities, and public and private organizations. Even if there are several recently published reviews in this area, e.g., Alessandrini et al. (2014), Cazzanti and Pallotta (2015), Tu et al. (2016) and Sidibé and Shu (2017), they do not provide an holistic and general overview of the state-of-the-art of the area of maritime anomaly detection.

The reviews presented in Cazzanti and Pallotta (2015), Tu et al. (2016) and Sidibé and Shu (2017) focus on the use of Automatic Identification System (AIS) data. The work presented in Tu et al. (2016) provides a broader perspective on how to exploit AIS for safety of seafaring in general, namely traffic anomaly detection, route estimation, collision prediction and path planning. Sidibé and Shu (2017) focus more closely on the use of AIS movement data for automatic anomalous maritime vessel behavior detection while Cazzanti and Pallotta (2015) discuss machine learning and data mining approaches for analyzing maritime vessel traffic based on AIS data as well, also tackling the interpretability of the results in real-world maritime situational awareness settings. The work presented in Alessandrini et al. (2014) is an introduction to maritime situational awareness by giving practical details on data integration and fusion, as well as how to how to extract contextual information of human-related activities from vessel positioning data. Therefore, in this paper, we provide a holistic view of maritime anomaly detection, covering not only methods for anomaly detection and different types of data, but also including systems with anomaly detection capabilities and related user aspects of maritime anomaly detection.

The work presented here started at the Workshop organized at the Joint Research Centre Ispra, entitled "Maritime Knowledge Discovery and Anomaly Detection Workshop," July 2016, joining academia and research centers, operational authorities and private sector working with maritime awareness related issues. Based on the discussions held there, we start a process of surveying the area of maritime anomaly detection in order to provide an overview of the field and outline research challenges.

Hence, this survey provides a comprehensive overview of the research carried 


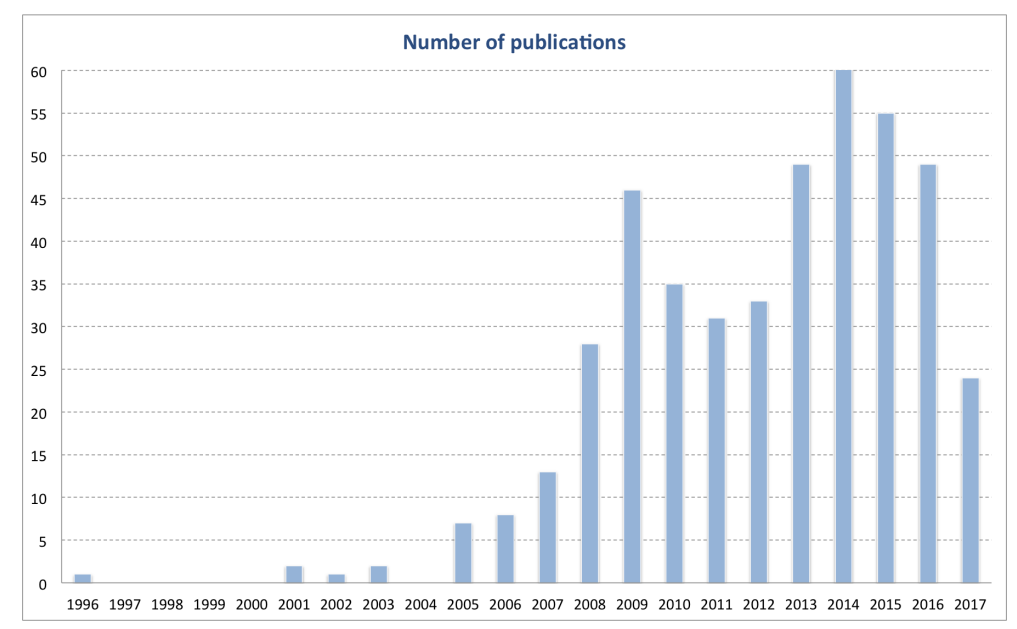

FIG. 1. Publications found during the search tackling maritime anomaly detection and related aspects per year.

out on maritime anomaly detection, in order to facilitate a better understanding of the different achievements regarding data, methods, systems and user aspects. For that, we present a taxonomy for classifying the vast number of publications found in this area, providing summaries of the works under each class. Based on this overview, we outline possible paths for future research.

\section{Organization}

This paper is organized as follows. First, section 2 provides a brief outlook to terminology related to anomaly detection, summarizes the method, search strategy, and keywords used, and presents the classification matrix used for the works found. The following sections, i.e., sections 3-6 provide a summary of the most relevant papers found in each category, namely, data, methods, systems and user aspects. Section 7 presents a summary of the challenges ahead identified for this domain, while section 8 concludes the paper.

\section{TERMINOLOGY, METHOD AND STUDY CATEGORIZATION}

\section{Terminology}

In this section, we define relevant terms for the survey. Maritime situation awareness is defined in COM-EU (2009) as the effective understanding of activity associated with the maritime domain that could impact the security, safety, economy, or environment [...]. The term anomaly is used in many domain areas with different meanings, thus, being a rather fuzzy concept which domain experts may have different notions of (Roy 2008). Anomaly is normally associated with terms of both a positive connotation, such as, normal (normalcy), usual, regular, typical, legal, interesting, and of a negative connotation, such as, abnormal, unusual, irregular, rare, deviation, strange, special, illegal, threating, exceptional, peculiar, outlier, atypical, inconsistent, etc. (Roy 2008). Moreover, the adjectives may refer to a situation, activity, behavior, event, happening, etc. In general, an 
anomaly always represents a deviation from normality. Data-driven anomaly detection algorithms consider anomalies, at least in the first stage, as anything that does not match the models that characterize normal data. For example, Portnoy et al. (2001) provide the following definition [a]nomaly detection approaches build models of normal data and then attempt to detect deviations from the normal model in observed data. Most of the published work within anomaly detection has its focus on automatic methods, thus following Portnoy et al. 2001's line of work. However, there is no definition of anomaly in Portnoy et al. (2001), nor what or which patterns are to be found in the data, making the evaluation of such methods a challenging task. Chandola et al. (2009) define anomaly as a pattern that does not conform to expected normal behavior; an anomaly detection approach, hence, defines a region representing normal behavior and declares any observation in the data which does not belong to this normal region as an anomaly. Many data mining techniques analyze data in order to find behavioral anomalies, which are defined as deviations from the normal behavior. For instance, Khatkhate et al. (2007) use the following definition within mechanical systems: [a]n anomaly is defined as deviation from the nominal behavior of a dynamical system and is often associated with parametric and non-parametric changes that may gradually evolve in time." Anomaly detection has been identified as an important group of techniques for detecting critical events in a wide range of data-intensive domains, where a majority of the data is considered normal (Chandola et al. 2009). As Ekman and Holst (2008) argue, anomaly detection says nothing about the detection approach, and it actually says nothing about what to detect." Perhaps the appeal of "anomaly detection" resides, both from a computational and human point of view, in the richness of its meanings, its complexity and in its vagueness.

\section{Method and analysis of the literature}

Since maritime anomaly detection has become an interdisciplinary subject, it required a literature search across several disciplines. We collected studies for this review from journal articles, conference and workshop papers, white papers, governmental reports and other review papers in the fields of computer science, security, maritime surveillance, military studies, maritime transportation, information fusion, information systems, cognitive science, etc. The library catalogs used during the search were Google Scholar, IEEE Xplore, Scopus, and ScienceDirect (more details can be seen in the appendix). We search for works with the main keywords maritime anomaly detection, and the search was restricted to 1996-2017. Fig. 1 shows the number of publications found per year, where it can be seen that there is a pick of research works published between 2013-2015. We excluded not-detailed descriptions of patents, duplicates (very similar works published in different venues or publication formats), publications/reports for which only an abstract or a slideshow were available, and even if considered under the category data, we excluded anomaly detection techniques applied over images. 


\begin{tabular}{|c|c|c|}
\hline CATEgory & DESCRIPTION \& SUB-TYPES & ENTRIES \\
\hline Data & $\begin{array}{l}\text { Data sources and data: AIS, radar, meteorological, } \\
\text { oceanographic } \\
\text { - Vessel tracking data and attributes } \\
\text { - Contextual Geographic Data } \\
\text { - Metadata }\end{array}$ & 31 \\
\hline Methods & Methods/techniques for maritime anomaly detection & 256 \\
\hline $\begin{array}{l}\text { Normalcy } \\
\text { extraction: } \\
\text { Normalcy } \\
\text { representation: }\end{array}$ & $\begin{array}{l}\text { - Data-driven approaches } \\
\text { - Signature-based } \\
\text { - Hybrid approaches } \\
\text { - Grid-based } \\
\text { - Vector-based } \\
\text { - Graph-based }\end{array}$ & \\
\hline Systems & $\begin{array}{l}\text { Systems and prototypes for maritime anomaly detec- } \\
\text { tion } \\
\text { - Requirements and design } \\
\text { - Systems } \\
\text { - Architectures } \\
\text { - Web-based services } \\
\text { - Frameworks }\end{array}$ & 116 \\
\hline User aspects & $\begin{array}{l}\text { Studies on user aspects, socio-technical systems, oper- } \\
\text { ators } \\
\text { - Characterization of maritime anomalies } \\
\text { - Socio-technical studies in maritime centers } \\
\text { - Requirements } \\
\text { - False alarm reduction, explanations, and anomaly } \\
\text { presentation } \\
\text { - Evaluations } \\
\text { - Visualization }\end{array}$ & 60 \\
\hline $\begin{array}{l}\text { Local and global } \\
\text { studies }\end{array}$ & $\begin{array}{l}\text { Studies particular areas, global concerns maritime } \\
\text { transportation }\end{array}$ & 32 \\
\hline $\begin{array}{l}\text { Motivation and } \\
\text { challenges }\end{array}$ & $\begin{array}{l}\text { Overall studies, motivation, current status maritime } \\
\text { surveillance }\end{array}$ & 18 \\
\hline Literature reviews & Surveys in the area of maritime anomaly detection & 5 \\
\hline
\end{tabular}

TABLE 1. Classification matrix for maritime anomaly detection related publications. 


\section{Study categorization}

Table 1 summarizes the categories used for classifying the works found. The broad categories selected are works focusing on (1) data and related aspects, (2) methods and techniques for maritime anomaly detection, (3) systems and (4) user aspects related to maritime anomaly detection. Under each broad category, we define several subcategories (see Table 1). We have used existing classifications for machine learning for classifying the methodological papers and inspiration from other broad surveys published on anomaly detection, in particular, Chandola et al. (2009). In the following sections, we provide a summary of each class.

\section{DATA}

This section summarizes works related to maritime data. Maritime situational awareness and anomaly detection data relate to vessels and their attributes (position, identification, history, etc.) and to contextual geographically-linked information. Vessel tracking data and attributes can be grouped into self-reporting (voluntary broadcast) or observation-based (active or passive sensors) depending on the way such data are acquired. Information registries and databases contain additional information about the vessel that is essential to build a solid awareness of what is happening at sea:

- Self-reporting positioning data are either transmitted by the vessel in their proximity for collision avoidance (Automatic Identification System $\mathrm{AIS}^{1}$ ) or sent using satellite communications to competent authorities for security and safety (Long Range Identification and Tracking LRIT $^{2}$ ), or for fisheries monitoring (Vessel Monitoring System $\mathrm{VMS}^{3}$ ). The technical specifications of such systems are regulated at national, international or global level. Such systems are characterized by different frequency of positioning update (ranging from a few seconds for AIS to a few hours for LRIT), quantity and types of vessels under regulation (e.g. EU fishing vessels exceeding $15 \mathrm{~m}$ for VMS in Europe) and, sometimes, spatial coverage (especially for AIS terrestrial receiving stations). The information content of self-reporting positioning data, in addition to position, velocity and other kinematic information, may also include voyage related and additional attributes related to the ship.

- Observation-based positioning data are instead collected by active or passive sensors. The detection capabilities of such sensors (e.g. spatial resolution, spatial coverage and update rate) vary depending on specific parameters (e.g. operating frequency, antenna size), environmental conditions (e.g. sea state, meteorologic and oceanographic data in the maritime

\footnotetext{
${ }^{1}$ Safety of Life at Sea (SOLAS) Convention chapter V regulation 19, carriage requirements for shipborne navigational systems and equipment (last amended in May 2006).

${ }^{2}$ Performance Standards and Functional Requirements for the Long Range Identification and Tracking of Ships. Resolution MSC.210(81); adopted on 19 May 2006.

${ }^{3}$ Commission Regulation (EC) No 2244/2003 of 18 December 2003 laying down detailed provisions regarding satellite-based Vessel Monitoring Systems.
} 


\begin{tabular}{ll}
\hline CATEGORY & DESCRIPTION AND SUB-CATEGORIES \\
\hline Vessel tracking data and attributes & $\begin{array}{l}\text { Data coupled to vessels from self-reporting } \\
\text { and observation-based sources. }\end{array}$ \\
& - Self-reporting positioning data \\
& - Observation-based positioning data \\
& - Information Registries and DB \\
Contextual Geographic Data & Vessel and operational context \\
& - Natural Environmental Data \\
& - Human related activities \\
Metadata & Quality, reliability, uncertainty, etc. associ- \\
& ated to data \\
\hline
\end{tabular}

TABLE 2. Maritime data, sources and categorization of works under this class.

domain) and target properties (e.g. size of a vessel, structure and orientation with respect to the point of observation). Examples of observationbased data include space-based Synthetic Aperture Radar (SAR) (e.g. Vespe and Greidanus (2012)), radars (e.g. Sermi et al. (2013)) or Maritime Patrol Aircrafts (MPA), Remotely Piloted Aircrafts (RPAs) sensors, electro-optical images (see Buck et al. (2008)) and video (see Shafer et al. (2015)). High Frequency (HF) radars (see Horstmann et al. (2010) and Braca et al. (2012)), passive radar (Griffiths and Baker (2005)), Over the Horizon radar (Langellotti et al. (2014)) and radiolocation of emissions (e.g. Papi et al. (2014)) are areas of research for maritime situational awareness and anomaly detection.

- Information Registries and Databases: such sources contain information linking a ship identifier as often used for tracking systems, to details about its construction, history, management, safety and security inspections. Such information is time unvarying or slowly changing if compared to the vessel position and motion. Vessel registries and databases therefore provide complementary data to tracking information, essential for maritime situational awareness and intelligence. After the inspection of a ship by Port State Control (PSC), according to PSC regional agreements (Paris MoU, Tokyo MoU, Black Sea MoU, etc.), if deficiencies are found, actions are taken such as informing the relevant Flag State, request the Master to rectify the deficiency before the departure or at next port of call, providing further insights into the behaviors of ships.

Contextual Geographic Data and Information: The context in which vessels move and operate has to be taken into account in order to accurately understand and monitor activities at sea. Indeed, behaviors that are apparently anomalous 
can often be explained if put into context. Two main categories of contextual geographic data and information are relevant to security and safety aspects of the maritime domain:

- Natural Environmental Data: Meteorological (wind fields and generic weather conditions) and oceanographic data sets (currents, bathymetry, shoreline etc.) are essential for safe navigation and risk assessment applications. In addition, such information can help in explaining alternative vessels routes.

- Human Related Activities: traffic management or routing systems, off-shore installations, port areas and facilities, risk or restricted areas etc. represent well-known information for both maritime safety and security. Such information is often difficult to source or access and sometimes can be found in the form of text (e.g., international regulations) that can be hard to keep updated. In case of unregulated routes or in open sea, this information is simply non-existent. Nonetheless, by analysing common collective behaviors of ships, it is possible to extract a wealth of elements of human related activities as demonstrated in the literature through knowledge discovery of vessel activities and historical data mining of vessel tracking information. This has led to the extraction of fishing footprint (Mazzarella et al. (2014), Natale et al. (2015)), main traffic routes (Pallotta et al. (2013d), Vespe et al. (2015)) and shipping densities (Alessandrini et al. (2014)) that can be used to identify the behaviour and activities "normality" against which deviations - and therefore anomalies - can be detected.

It is worth noting that there is no ideal sensor or technology that fits all surveillance applications: all the data produced have intrinsic limitations, especially if used beyond the purpose for which the data are collected. In addition, there are specific requirements that cannot be properly fulfilled by any of the available data as, for example, for the detection of small boats in wide areas, where vessels need to be detected and persistently tracked in open sea. The definition of such requirements should drive the resource investment for additional data (see Horn et al. (2016)). Nevertheless, system integration and data fusion can significantly overcome the limitations of single self-reporting and observation-based systems (see Carthel et al. (2007) and Vespe et al. (2008)).

Metadata for most of the above-mentioned sources are somewhat unavailable: although the understanding of the technology and of the relevant accuracy information can be straightforward, the quality of the data may vary depending on a number of factors. As an example, the quality of AIS coverage may be due to environmental conditions and varies depending on the geographic location of the receiver. This is mainly because vessel tracking systems were originally developed in a strict application-oriented way. Their use for knowledge discovery, data fusion, and anomaly detection is becoming nowadays an emerging field of 
analysis, and the effort to systematically produce metadata may be requested in the near future.

\section{METHODS}

A large number of the found works were classified in the category "methods and techniques for maritime anomaly detection". There are clearly several ways of classifying the methods for detecting anomalies in maritime traffic. For instance, algorithms used in the detection of intrusions are traditionally classified into three main groups: data-driven approaches, signature, or hybrid. Systems based on anomaly detection schemes (data-driven approaches) look for abnormalities in the traffic, assuming that something abnormal is probably suspicious. They generally estimate the degree of deviation of the test trajectories from the learned normalcy. The usability of this approach seems easier to be applied on a larger scale to gain efficient classification performances and detect different types of anomalies. The majority of works found in this area are under this category, in particular, unsupervised solutions. Signature-based approaches look for predefined patterns in the data such as specific trajectory evolutions (e.g., loitering vessels, U-turns or multiple loops such as to avoid an obstacle), whereas hybrid solutions combine data and knowledge-driven approaches. These latter approaches (signature-based) look promising for real-time applications since they do not explicitly rely on historical information, although the behavioral modeling is generally helped by the analysis of similar anomalous behaviors seen in the past. In Kazemi et al. (2013) a large list of anomalous behaviors and the list of events corresponding to those anomalies are listed out, along with a validation study using open data.

It is challenging to derive an exhaustive anomaly detection taxonomy, due to the variety of anomalies in the maritime domain. Generally, the maritime traffic normalcy is shaped as historical vessel patterns-of-life and is used as baseline information in order to detect critical events. Based on which features are considered in the definition of the critical events, different anomalies can be detected, and, hence, different anomaly detectors can be tailored and the normalcy representation is informed by these.

Complex events require a combined approach with either a signature-based anomaly detector or a combination of modular individual anomaly detectors, each one addressing a single-type anomaly.

In the analyzed literature, the most frequently detected anomalies can be classified as follows:

- positional anomalies, i.e., vessels traveling off the main route in unusual areas:

- in Laxhammar (2008), Laxhammar et al. (2009) and Laxhammar and Falkman (2010) anomalies are searched starting from sea traffic shaped as a four-dimensional feature space (i.e., momentary position in latitudinal and longitudinal coordinates and the velocity 
in latitudinal and longitudinal directions of vessels). They consider that four-feature variable as constituting a single data vector and statistically model it using a joint probability density function (pdf);

- in Mascaro et al. (2010) and Mascaro et al. (2014) different anomalies are searched. The positional ones are somehow data-related such as "travel over land".

- in Willems et al. (2009), Willems et al. (2010), and Willems et al. (2013) the authors highlight anomalies caused by vessels which sail outside of the designated shipping lanes, such as a cargo vessel which goes off-route to resupply ocean platforms in the area under investigation;

- in Lane et al. (2010) and Lane (2011) deviations from a standard route are detected as a sign of positional anomalies;

- in C.Zor (2017) anomalous ferry tracks are highlighted;

- contextual anomalies e.g., vessels whose deviating behavior is directly correlated to the context;

- e.g., Hayes and Capretz (2015) discuss anomalies correlated to timerelated concepts such as seasons, days of the week, workdays versus holidays;

- e.g., a tanker traveling on a ferry route;

- kinematic anomalies:

- vessels showing a wrong or unusual course over ground such as sailing in the opposite direction on an established route, as in Kazemi et al. (2013), Mascaro et al. (2010), Mascaro et al. (2014) and Anneken et al. (2015a)

- high-speed or low-speed vessels as in Laxhammar et al. (2009) and Laxhammar (2008)

- course, velocity and U-turn anomalies as discussed in Keane (2017);

- instantaneous stops and turns as described in Patroumpas et al. (2017);

- complex anomalies whose detection requires an ensemble of anomaly detectors to capture specific behaviors:

- in Kowalska and Peel (2012) complex anomalies such as vessels engaged in drug smuggling, people smuggling and terrorism are classified;

- loitering vessels whose detection relies on the use of speed gating algorithms (comparing the actual and the estimated future subsequent displacements of vessels as discussed in Cazzanti and Pallotta 
Pre-peer reviewed version.

Riveiro M., Pallotta G., and Vespe M. Maritime anomaly detection: A review. WIREs Data Mining Knowl Discov.

Advanced Review. 2018;e1266.

\begin{tabular}{ll}
\hline CATEGORY & EXAmPle OF MOST POPUlAR METHOD Within CATEGORY \\
\hline Parametric & Gaussian Mixture Models \\
Non-Parametric & Kernel Density Estimation \\
Clustering & Density-based spatial clustering of applications with noise \\
\hline
\end{tabular}

TABLE 3. Methods for Maritime Anomaly Detection: how normalcy is extracted

(2015);

- spoofing behavior as in Katsilieris et al. (2013a) and Mazzarella et al. (2017).

- data-related anomalies:

- in Dobrkovic et al. (2018) incomplete trajectory data are classified as anomalies;

In this review, the first level of classification concerns how normalcy is (1) extracted (Table 3), and (2) represented (Table 4).

Classification of the anomaly detection methods: ways to extract the normalcy.

This section describes the normalcy extraction, which is a prerequisite to the application of any anomaly detection method. The way in which normalcy is derived, starts from the definition of the anomaly detection capability both in terms of the types of anomalies of interest and of the desired accuracy. A first way to distinguish different normalcy extraction methods is on the basis of the amount of 'external information' injected into. Based on that, we can speak of supervised, unsupervised and semi-supervised anomaly detection methods.

Table 3 summarizes the different methods to extract normalcy for maritime anomaly detection.

Due to the characteristics of the anomaly detection problem, i.e., its complexity, its ill-defined nature, and the lack of labeled data for the anomalous class, the majority of the methods to extract a normal representation of the data are unsupervised, i.e., unsupervised anomaly detection techniques detect anomalies in unlabeled datasets, based on the assumption that the majority of the instances are normal. Most of the published work in this field generates a maritime traffic normalcy from historical self-reporting positioning data (e.g., AIS).

Statistical anomaly detection methods:

- Parametric methods: some statistical generative models are applied to the historical traffic. Gaussian Mixture Models (GMM) are a very popular choice for approximating the (unknown) multivariate probability density functions (pdfs) of normal vessel traffic. One of the most critical points is the selection of the number of components in the mixture (generally an 
Expectation-Maximization (EM) algorithm is preliminarily used) and most of the times these methods are computationally demanding and cannot be easily applied to a large area; The general aim of this approach is to cluster the data in a multi-dimensional feature space, where the features of the trip are variables such as longitude and latitude, heading and speed. In this way, multivariate pdfs are handled.

- Non-parametric methods: Kernel Density Estimation (KDE) of traffic normalcy is a popular method which derives a non-parametric model of traffic normalcy. One delicate step is the selection of the kernel (most papers adopt a Gaussian kernel) and of an optimized bandwidth. An adaptive bandwidth selection is analyzed in Laxhammar et al. (2009) and used in Pallotta et al. (2013b). An adaptive KDE is used to derive a normal traffic model in Ristic et al. (2008) and then particle filters are used to predict position of vessels based on that derived density.

- Clustering methods: K-means is a popular 'distance-based' clustering method. Its popularity is due to the fact that it is easy to implement. Its main drawback is the selection of the number of clusters, which, as previously discussed, can be optimized by using an EM algorithm. An example of the maritime data clustering based on K-means can be found in Vespe et al. (2012); recently Density-based spatial clustering of applications with noise (DBSCAN) methods have become very popular for their convenient properties as compared to k-means: they are density-based (which is a convenient property for the maritime data), they do not require to specify the number of clusters, they have the ability to derive arbitrarily shaped clusters and they incorporate by-product the classification of the noise points; examples of such applications in the maritime domain can be found in Pallotta et al. (2013c), Arguedas et al. (2014), Pallotta et al. (2013a) and Pallotta and Jousselme (2015). Liu et al. (2015) discusses an 'ad-hoc' similarity metric for trajectory partitioning and segment clustering, which can be seen as a DBSCAN version for trajectory clustering.

Depending on the way the points from trajectories are processed by the anomaly detectors, methods for anomaly detection can be distinguished into two main classes:

- point-based anomaly detection methods, which highlight individual anomalous points, as in Kowalska and Peel (2012) and Le Guillarme and Lerouvreur (2013)

- trajectory-based anomaly detection methods, which receive as input entire trajectories and automatically classify them as anomalous or not, as in Laxhammar (2011) and de Vries and van Someren (2013).

Generally, these anomaly detection methods are informed by the way the normalcy is constructed, being the former ones derived from point-based clustering methods (as K-means and DBSCAN), the latter ones related to the clustering 


\begin{tabular}{ll}
\hline CATEGORY & DESCRIPTION \\
\hline Grid-based & Design a grid over the discretized vessel locations/kinematic features \\
Vector-based & Use similarity metrics between complete/evenly spaced trajectories \\
Graph-based & Derive a topological/topographical network of shipping flows \\
\hline
\end{tabular}

\section{TABLE 4. Anomaly Detection Methods: how normalcy is represented}

of entire trajectories based on similarity measures. This is strictly linked to the problem of real-time anomaly detection which is still hard to tackle. As a matter of fact, most of the algorithms available in literature are designed for off-line anomaly detection in trajectories. Hence, they are based on the assumption that the anomaly classification is performed after the entire vessel trajectory has been observed. This is an evident drawback for surveillance applications since it delays the alert on anomalous events, thus delaying the necessary actions to be taken. In contrast, algorithms for sequential anomaly detection allow detection in case of incomplete or partially observed trajectories (e.g., trips, tracklets or trajectories), e.g., real-time detection of anomalous trajectories as they evolve.

\section{Classification of the anomaly detection methods: ways to represent the normalcy.}

Another way to classify the anomaly detection methods is based on the representation of the normalcy. Table 4 reports the three main categories for normalcy representation in which the works found in literature can be classified.

Grid-based techniques usually divide the monitored area using a spatial grid whose cells contain the motion properties (e.g., location, heading and speed) of the crossing vessels. This approach is fast enough to detect simple point anomalies but it suffers from computational burden for large monitored areas. Moreover the selection of the optimal cell size is critical and often not straightforward and the adoption of a grid structure looses the spatio-temporal correlation of vessel tracks, making difficult or impossible the detection of structured anomalies (e.g., start/stop events). The grid-based methods have been considered effective only for small area surveillance and the computational burden was regarded as its limitation when increasing the scale. Some examples of such a representation can be found in Bomberger et al. (2006) where a discretized representation of vessel locations is reported and in Ristic (2014) where a speed-based grid of vessel activity is presented.

Vector-based methods are based on spatio-temporal data mining. These techniques start from the reconstruction of vessel tracks and trajectories by using start, end and intermediate waypoints and then use similarity metrics to cluster the trajectories. One drawback is that they often require complete or evenly spaced trajectories as input data. This can be an issue when the refresh rates from different sensors can generate intermittent, incomplete and unequal length trajectories. This problem is addressed in the following papers: Etienne et al. 
Pre-peer reviewed version.

Riveiro M., Pallotta G., and Vespe M. Maritime anomaly detection: A review. WIREs Data Mining Knowl Discov.

Advanced Review. 2018:e1266.

\begin{tabular}{ll}
\hline CATEGORY & DESCRIPTION AND SUB-CATEGORIES \\
\hline Requirements and design & Requirements specification and system design \\
Systems & Maritime anomaly detection systems, commercial and \\
& research-based proof-of-concepts \\
& - Data-driven based systems \\
& - Rule-based based systems \\
& - Hybrid (compound solutions) \\
& Focus on underlying architectures and infrastructure \\
Architectures & We-based solutions, distributed systems \\
Web-based services & Combination of methods, anomaly detection modules \\
\hline
\end{tabular}

TABLE 5. Classification of works on maritime anomaly detection systems.

(2011), Pallotta et al. (2013a), Pallotta et al. (2013c), Pallotta et al. (2013b), de Vries and van Someren (2013) and Arguedas et al. (2015). These methods look more suitable for maritime anomaly detection since they enable the sequential classification of track points with a scoring which can be updated as soon as a new vessel report, along with the monitored track, is received.

Graph-based methods are a relatively recent approach in maritime applications. They derive activity nodes and waypoints and then generate the network of traffic flows. In the book Ait-Mohand et al. (2015) and subsequent works, the concept of maritime networks and the related connectivity of maritime nodes (i.e., ports and terminals) is discussed. This is one of the most extensive investigations of maritime networks found in the literature. The traffic flows are represented between a pre-defined set of nodes in a graph-based fashion and the networks are derived as a topographical map of the shipping connections. In Caschili et al. (2014) the analysis of shipping networks is analysed via a network representation, where the main indicators of network connectivity are derived. However, this network-based representation is useful for descriptive purposes of the shipping activity but provides little room for classification and anomaly detection. A more promising representation based on geographical networks is discussed in Arguedas et al. (2014), Arguedas et al. (2015) and Arguedas et al. (2017) where a simplified and structured picture of the maritime activity at sea is derived and some examples of anomaly detection are provided. The strategy presented in these works first derives the points of interest using a density-based approach (i.e., DBSCAN); then it estimates the average paths between them by using the actual in-between vessel tracks connecting the points and derives the synthetic routes. The advantage is the reduction of the storage requirements for the maritime routes, but without loosing the real geographical mapping of the connections between the nodes. The representation of the intermediate paths (called synthetic routes) enables the detection of both positional and kinematic anomalies. The authors show that the reduced representation still provides good results in terms of classification. 


\section{SYSTEMS}

This section provides a summary of works that present systems, frameworks and related issues for maritime anomaly detection found during the literature survey. The number of publications found under this category is vast (more than 100 works), and the systems found go from very preliminary frameworks to fully functional prototypes to commercial systems. We present major works and examples on complete systems, frameworks, architectures and web-based services. Based on the type of anomaly detection method, we further present data-driven, signature -based or hybrid solutions.

Systems based on data-driven anomaly detection schemes look for abnormalities in the traffic, assuming that something that is abnormal is probably suspicious. Such detectors are based on what constitutes normal behavior and what percentage of the activity we want or are allowed/willing to flag as abnormal (thresholds). Kraiman et al. (2002) present a statistical parametric anomaly detector processor, that exploits multisensor tracking and surveillance data to identify interesting events. The authors demonstrate the detector within a VTS environment, and the detection approach is a statistical parametric method, based on a combination of Self-Organizing Maps and Gaussian Mixture Models.

Many of the anomaly detection capabilities implemented in real maritime control centers are rule or signature, -based systems. Such systems allow an operator to create simple rules that will trigger an alarm (e.g., IF jvessel in shallow waters $;$ THEN jdanger of grounding $i)$. Examples of this type of systems are Roy (2008, Fooladvandi et al. (2009, Roy (2010, Edlund et al. (2006) and Osekowska and Carlsson (2015). These works also relate to detection of anomalous behavior based on reasoning capabilities exploiting ontologies, see, e.g., Roy and Davenport (2010) and Vandecasteele and Napoli (2012b, Vandecasteele and Napoli (2012a).

An example of a hybrid system, i.e., a combination of both data-driven and knowledge-based methods, is SeeCoast Rhodes et al. (2006, Rhodes et al. (2007b). SeeCoast extends the detection capability of the learning-based pattern module based on Rhodes research (BAE Systems) on the problem of learning normal vessel motion patterns (see work on development of such methods in Rhodes et al. (2005, Rhodes et al. (2007c, Bomberger et al. (2008, Rhodes et al. (2007a)). SeeCoast applies rule-based and learning-based pattern recognition algorithms to alert illegal, threatening and anomalous vessel activities. The system takes realtime tracking information and uses continuous on-the-fly learning that enables the concurrent recognition of patterns of current motion states.

Discussions on requirements or which properties maritime systems should have in the future are presented in Hiemstra (2008), through the following problem areas: (1) asset and vessel protection, (2) traffic management, (3) maritime surveillance and (4) awareness, coordination and control. However, this type of works is very scarce in the literature. The design of anomaly detection systems is very rarely tackled in this field as well, even if there are some exceptions, such as Colmanta and van Vuurena (, Singh et al. (2016). 
As mentioned earlier, there are many systems presented in the literature for maritime anomaly detection, e.g., Rothkrantz and Scholte (2013, Malhotra et al. (2011, Lei et al. (2017, Ray et al. (2015, St-Hilaire and Hadzagic (2013, Riveiro and Falkman (2009, Kanellopoulos et al. (2015, Wen et al. (2014, Morel et al. (2008, Fridman et al. (2017). Research on tracking and detection of anomalous behavior for container traffic has also resulted in a series of works that analyzed these type of data. An example of this kind is "ConTraffic" (Varfis et al. 2011). Web-service based systems for maritime situational awareness are Dimitrova et al. (2013a, Dai and Liu (2015), while a more focus on architectural issues and solutions for providing maritime awareness are presented in Zissis et al. (2015, Stryna et al. (2016).Several are the commercial ventures and real-world applications found with strong ties to research in this area, even if they are not always well-documented, e.g., Sjbasis (Sweden), Oversee (Portugal), ABM (EMSA), AMIS (Australia), MSA Brite (NATO), etc.

Finally, the following articles were classified under the category framework, since they present the basis, structure or processes for developing a anomaly detection system, Li et al. (2007, Avram (2012, Pallotta et al. (2013d, Vandecasteele et al. (2013, Shahir et al. (2014, Jaszewski et al. (2015) and Lei (2016).

\section{USER ASPECTS}

The complexity and volume of sea traffic require a form of traffic control, in order to optimize traffic streams, protect the environment and guarantee safety. These tasks are usually done by services such as Vessel Traffic Services (VTS). For instance, coastal VTS monitor traffic in the corresponding areas, while port VTS are primarily concerned with vessel arrivals/departures to and from harbors. An example of a complete socio-technical study of VTS operators is presented in Brdje (2012). Other authorities that are interested in the surveillance and monitoring of the sea waters are coastguards, military authorities, border protection, maritime administration, police, environmental agencies, etc. Personnel that provides these services have to their disposal, as seen under section 3, various sensors and sources of information, radar data, AIS, VHF radio, closed circuit TV cameras, maps, administrative information (logistics), data bases with historical information, meter/hydro information, etc. Unfortunately, this heterogeneous data is not normally integrated into a unique surveillance system, and therefore, there might be a high cognitive load on operators monitoring sea traffic, since they need to control many systems and sensors while trying to detect conflict situations. Due to that, user aspects related to the use of maritime anomaly detection methods or systems including anomaly detection capabilities have grown in research interest in recent years. Under this category, we found papers with a very disparate focus that tackle different challenges for the users of these systems, e.g., the reduction of false alarm rates, the visualization of maritime traffic, anomalies or normalcy models, empirical evaluations of such systems, etc.

The reduction of false alarm rates is the focus of several studies, such as Rossi et al. (2014, Radon et al. (2015, Radon (2015); they propose different 


\begin{tabular}{|c|c|}
\hline CATEGoRy & DESCRIPTION AND SUB-CATEGORIES \\
\hline $\begin{array}{l}\text { Characterization of maritime } \\
\text { anomalies }\end{array}$ & Types and classes of anomalies, rules \\
\hline Requirements & $\begin{array}{l}\text { Requirements specification and design of maritime } \\
\text { systems }\end{array}$ \\
\hline $\begin{array}{l}\text { Socio-technical studies in } \\
\text { maritime centers }\end{array}$ & $\begin{array}{l}\text { Operators analytical and surveillance tasks, infor- } \\
\text { mation sharing between authorities, policies }\end{array}$ \\
\hline $\begin{array}{l}\text { False alarms, explanations } \\
\text { and anomaly presentation }\end{array}$ & $\begin{array}{l}\text { Studies on false alarm reduction, how to provide } \\
\text { explanations and present outliers to operators }\end{array}$ \\
\hline Evaluations & User empirical evaluations \\
\hline Visualization & $\begin{array}{l}\text { How to present maritime traffic and anomalies to } \\
\text { operators } \\
\text { - Interactive Visualization } \\
\text { - Visualization of traffic and density maps } \\
\text { - Visual analytics: applicability, systems and geo- } \\
\text { visual analytics } \\
\text { - Evaluation }\end{array}$ \\
\hline
\end{tabular}

TABLE 6. Classification of user aspects works on maritime anomaly detection.

techniques for the reduction of high alarm rates using, from adding contextual information for verification in Radon et al. (2015, Radon (2015) to particular solutions to overcome glint in image analysis for airborne hyperspectral detection of anomalous objects at sea (Rossi et al. 2014).

Knowledge elicitation from experts, rules formulation or just in general, extracting knowledge from domain experts for classifying anomalies are the focus of works such as Nilsson et al. (2008, Roy and Davenport (2009). Both works present a useful classification of maritime anomalies from maritime experts.

One challenge associated with the presentation of anomalies to users regards providing associated explanations for the causes and context of such anomalies. This challenge is tackled in, e.g., Helldin and Riveiro (2009) and Dragos (2016), which try to provide causes for anomalies detected using Bayesian Networks and semantic integration from information retrieval techniques, respectively. This is, however, quite an important challenge in this area, which has received very little attention. To provide efficient support for operators, there are two examples of studies that illustrate the typical analysis tasks the operators carry out on a daily basis, Riveiro et al. (2009a) and Vatin and Napoli (2013b).

Other works that were classified under user aspects are requirements for maritime surveillance systems, e.g., Lee et al. (2014), bridging the gap between policy and technology, Galdorisi and Goshorn (2005), information sharing and collaboration between maritime authorities White and Semy (2010, MacLeod and Wardrop (2015), socio-technical studies Nuutinen et al. (2007, Brdje (2012), and particu- 
lar challenges for operators of AIS satellite systems, e.g., Nordmo Skauen et al. (2013).

Within this review, we found, surprisingly, very few works that tackle any evaluation of maritime anomaly detection systems with users. Very few exceptions were found, Riveiro and Falkman (2010, Riveiro (2014), but, unfortunately, they include very few real-world operators.

A large number of publications under user aspects in maritime anomaly detection focus on the visualization of maritime traffic. One of the best examples is the visualization of vessel density and moving objects at the harbor of Rotterdam, The Netherlands, presented by Willems et al. (2009, Willems et al. (2013), visualization of container movements, e.g., Dimitrova et al. (2013b), visualization of normalcy models, Riveiro and Falkman (2009) and other representations related to finding or representing anomalies in traffic, for instance, Osekowska and Carlsson (2009). The initial works considering interactive visualization as means for supporting monitoring the traffic and the detection of anomalies, such as Riveiro et al. (2008, Dimitrova et al. (2014, Lavigne (2014a), evolved more recently on the use of Visual Analytics. Visual Analytics has been defined as "the science of analytical reasoning facilitated by interactive human-machine interfaces" (Thomas and Cook 2005);" visual analytics combines automated analysis techniques with interactive visualizations for an effective understanding, reasoning and decisionmaking on the basis of very large and complex datasets" (Keim et al. 2010). The applicability or use of Visual Analytics for maritime surveillance is suggested and discussed in several works, for instance, Lavigne and Gouin (2011, Varga and Lavigne (2016, Davenport (2009, Riveiro (2013) and visual analytics systems or processes for maritime anomaly detection are presented in Lavigne (2014b, Riveiro et al. (2009b, Nandan et al. (2013, Malik et al. (2014, Hanna (2015, Wang et al. (2017). Similar works focus on the geographical and temporal nature of the data, and very often, those are presented within geovisual analytics, e.g., Vatin and Napoli (2013a, Vatin and Napoli (2013c). More specific work on the visualization of outliers appears in Etienne et al. (2011) while integrating visual information in existing VTS systems is the focus of Bloisi et al. (2015, Bloisi et al. (2017).

\section{CHALLENGES}

As highlighted by Martineau and Roy (2011), anomaly detection in the maritime domain was identified by the operators/analysts of the operational community as an important aspect requiring research and development. During our review, we have collected references to challenges, future works and unresolved issues that can constitute promising directions for further research in maritime anomaly detection. This section summarizes such challenges, presenting them under the aforementioned categories: data, methods, systems, and user aspects, even if some of the challenges clearly relate to each other and are intertwined. The challenges outlined were complemented with other reviews and published challenges in this field, for instance, Boraz (2009, Roberts (2014, Moreira et al. 
(2014, Tu et al. (2016) and Sidibé and Shu (2017).

Data One repeated challenge throughout the literature revised under this category relates to integrating and fusing heterogeneous data and information in an integrated situation picture. There are numerous papers on maritime anomaly detection published in the area of information fusion, but still, the disparate nature of the data and information, the velocity at which is generated, their differences in type, sampling frequencies, veracity, etc. still hinders the real-time construction of a reliable, integrated and complete situation picture that supports maritime situation awareness. Moreover, to our surprise, even if we found some works, e.g., Wang et al. (2014), not too many works were found on the use of Big Data technologies in this domain, as highlighted by (Sidibé and Shu 2017) as well. Generally, the need of handling large amounts of complex data for an integrated maritime picture is stressed by other authors, e.g., Graham (2009, Balci and Pegg (2006, Braca et al. (2015). Some authors further specify that these technologies are already available, nevertheless, the challenges lie more in their cohabitation (Martineau and Roy 2011). An update on the use of Big Data for maritime knowledge discovery associated with the detection of safety and security threats is summarized in Garnier and Napoli (2016).

The exploitation and use of contextual information for improved situation awareness is quite an active area of research for fusion systems. Results in this area are crucial in the advancement of research in maritime anomaly detection, for instance, for reducing false alarm rates. Even if we have seen very interesting works exploiting context in this domain, e.g., Pallotta et al. (2014, Snidaro et al. (2015a), and there is a growing interest in this topic, more research is needed on how to represent context for maritime anomaly detection.

There are other persistent challenges associated to the data common to other data-intensive domains, e.g., those that we can consider meta-data issues, for instance, uncertainty, reliability, missing data, integrity, lack of quality, coverage problems, dirty data, cluttered environments for radar, AIS spoofing, etc. Uncertainty management is the focus of several of the works found for maritime anomaly detection under methods, e.g., Hunter (2009, Jousselme and Pallotta (2015, Snidaro et al. (2015b), however, some interesting suggestions to overcome this type of problems relate to the use of other data sources. For instance, the reliability and coverage of the AIS reports could be monitored from radar data, as addressed in Katsilieris et al. (2013b, Braca et al. (2015). Different sensors can complement each other, as addressed by Boraz (2009), for overcoming AIS spoofing and coverage, radar range, lack of images that cover the entire globe, etc. Finally, time difference of arrival from multiple AIS receivers (Papi et al. (2014)) or the signal strength at the receiver can be used to detect spoofing or on-off switching (Mazzarella et al. (2016, Mazzarella et al. (2017)).

Other issues related to data concern sharing data between different authorities and organizations. This is a persistent problem, not unique of the maritime domain, but very relevant in this area. Problems related to cooperation and 
data sharing between organizations are described bellow under systems and user aspects related challenges.

Methods Several of the challenges related to methods are parameter initialization, long-term learning strategies and normalcy models adaptation. Algorithms for anomaly detection require parameter initialization, e.g., thresholds, number of layers, etc. that normally have a great influence on the performance of anomaly detection methods. Support for such parameter tunning and the analysis of its consequences should be provided. Long-term learning strategies (how to cope with large data sets) is a persistent problem in data-intensive environments such this one, and moreover, most approaches have difficulties with normalcy model adaptation Brax (2011). If no data pruning strategy is used, online learning and anomaly detection may become intractable due to the size of the training sets. In general, very few studies were found that tackle scalability problems and realtime anomaly detection, issue also highlighted by (Sidibé and Shu 2017). We also observed a lack of studies regarding feature extraction and finding relevant features in high-dimensional maritime data sets.

Moreover, there is a lack of annotated and open datasets to evaluate maritime anomaly detection methods (Anneken et al. 2015b), as well as a lack of representative tests for performance evaluation of anomaly detection methods with labeled data.

Systems Several of the persistent challenges under systems relate to aspects already tackled under data and methods, for example, real-time functionality and scalability problems associated with the vast amounts of maritime complex data. Incompatibility between maritime systems and a need for integration is another common problem in this domain, as stressed by, e.g., Balci and Pegg (2006, Olson (2006). Sharing information and processes between systems over regional, local and country borders, and at various levels and among different organizations is desirable Graham (2009, Martineau and Roy (2011), and more work needs to be done in this regard.

Based on the work found under systems, we concluded that little work had been devoted to requirement specification for future maritime anomaly detection systems, as well as very few papers include any detailed user-centered design approach or empirical evaluation of the proposed systems or proof-of-concepts. Even if some exceptions exist, we think that the work in system development for supporting maritime anomaly detection has been driven from a technological perspective, and future designs would require greater involvement of the final users, to overcome some of the challenges found under user aspects. For instance, since anomaly detection is an ill-defined problem, we need systems that allow expert input, to improve anomaly detection performance and reduce false alarms rates. Furthermore, we need system transparency, in order to support user understanding of the system inner workings and limitations, for supporting trust calibration. 
User aspects Even if there is a growing interest in studies related to the user and the use of anomaly detection methods and systems, there are still persistent research challenges under user aspects. Challenges highlighted here relate to, as seen under systems, the insertion of expert knowledge in the anomaly detection process, the interpretability of the anomaly detection process when needed, to support calibrated levels of trust, and in general, finding efficient humanmachine collaboration. Moreover, how to initialize parameters to the anomaly detection process, how to provide explanations to operators and how to update and improve normalcy models by operators, are still unresolved issues. A related discussion here is, of course, the different types of users and backgrounds of maritime anomaly detection systems, and what kind of knowledge and background is required to set input parameters, update models or interact with the learning phases of the methods. These are, however, common challenges shared with other domains that use artificial intelligence (AI) and machine learning solutions. The need for transparency in ML systems to support interpretability, understandability, and trust has been highlighted recently by multiple authors from disparate disciplines, both from the AI and Human-Computer Interaction communities, e.g., (Zhou et al. 2013; Amershi et al. 2014; Rudin 2014; Ribeiro et al. 2016; Sacha et al. 2016). There is clearly a trade-off between accuracy and transparency, but when the problem is complex or ill-defined as anomaly detection, a combination user-machine is normally beneficial.

The manual operation of surveillance systems may be not efficient due to fatigue, stress, overload and the limited ability of the operators performing certain tasks (Moreira et al. 2014). In order to provide more support, a taxonomy of analytical tasks for operators working with surveillance systems at maritime traffic control services needs to be developed (Riveiro 2011), as well as taxonomies of anomalies, singular and collective. Other aspects related to maritime anomaly detection methods that have a great impact on operators workload and trust are the high number of false alarms mainly connected to unsupervised learning techniques, therefore the reduction of false alarms rates is a major improvement needed in current maritime anomaly detection methods.

Complete socio-technical, organizational and cognitive studies will reveal possible particularities that may affect working procedures and tasks and how to improve them. Generally, one of the most persistent challenges for maritime surveillance is associated to overcoming cultural, political and legal restrictions that hamper the ability of various countries to share information and join efforts Boraz (2009). There are several authors that claim that a maritime interagency task force will allow a global maritime domain awareness. The cooperation between agencies is also encouraged, overcoming policy restrictions and restricted access to certain data and information due to different security levels and information classification schemes (Martineau and Roy 2011). Finally, the capability to detect anomalous behavior allowing for interdiction and response should have minimal impact to legitimate commerce (Olson 2006). 


\section{CONCLUSIONS}

In this paper, we presented a review of studies focusing on maritime anomaly detection since 1996 to 2017. The large amount of works found were categorized considering their main contribution into methods, data, systems and user aspects of maritime surveillance (see details in table 1). This provided a more structured way of looking at the research in this domain, which also showed the interdisciplinary nature of the work in this area. We analyzed the findings from the studies under review summarizing their contributions for each category. Finally, for each class, we identified challenges and promising directions for further research.

In conclusion, the above literature review showed the wide range of scientific areas needed for a coherent development in the area of maritime anomaly detection. The core of the work carried out has its focus on methods for anomaly detection, and within this class, the majority of the methods extract a normal representation of the data in an unsupervised manner with unlabeled datasets. Since anomaly detection is normally a complex problem, there have been and still are many attempts to improve current algorithms with increased performance, in many occasions, finding combinations of various techniques. The majority are still off-line methods, and more work is needed finding solutions for real-time surveillance applications. Other challenges ahead regarding methods relate to the scalability of the solutions and the adaptability of the normalcy models. Adding contextual information and meta-data aspects were also highlighted in connection with the challenges associated with the data employed in this domain. We believe there is a huge opportunity to perform other important studies that focus on the design, use, and evaluation of systems that embed anomaly detection capabilities by operators and expert personnel. Along these lines, the need for more transparent, interpretable and explainable machine learning-based systems has been highlighted recently by multiple authors from disparate disciplines, e.g., from the AI and HCI communities in Amershi et al. (2014), Rudin (2014), Ribeiro et al. (2016) and Burrell (2016). Developments in the intersection of these disciplines will contribute to solving challenges within maritime anomaly detection related to, for example, the insertion of expert knowledge, true user-machine collaboration in complex cases and support for a calibrated level of trust of future surveillance systems.

\section{ACKNOWLEDGEMENTS}

This study has been partially supported by the Swedish Knowledge Foundation, project NOVA (2014/0294).

\section{REFERENCES}

Ait-Mohand, K., Alincourt, E., Barthelemy, M., Bouveyron, C., Bretagnolle, A., Coche, M.-A., Cuyala, S., Devogele, T., Didier, L., Ducruet, C., et al. (2015). Maritime Networks.

Alessandrini, A., Argentieri, P., Alvarez, M. A., Barbas, T., Delaney, C., Arguedas, V. F., Gammieri, V., Greidanus, H., Mazzarella, F., Vespe, M., and others (2014). 
"Data driven contextual knowledge from and for maritime situational awareness." Context-Awareness in Geographic Information Services (CAGIS 2014), 39.

Amershi, S., Cakmak, M., Knox, W. B., and Kulesza, T. (2014). "Power to the people: The role of humans in interactive machine learning." AI Magazine, 35(4), 105-120.

Anneken, M., Fischer, Y., and Beyerer, J. (2015a). "Evaluation and comparison of anomaly detection algorithms in annotated datasets from the maritime domain." 2015 SAI Intelligent Systems Conference (IntelliSys), 169-178 (Nov).

Anneken, M., Fischer, Y., and Beyerer, J. (2015b). "Evaluation and comparison of anomaly detection algorithms in annotated datasets from the maritime domain, comparative." IntelliSys 2015 - Proceedings of 2015 SAI Intelligent Systems Conference, Institute of Electrical and Electronics Engineers Inc., 169-178.

Arguedas, V., Pallotta, G., and Vespe, M. (2017). "Maritime Traffic Networks: From Historical Positioning Data to Unsupervised Maritime Traffic Monitoring." IEEE Transactions on Intelligent Transportation Systems, PP(99), 1-11.

Arguedas, V. F., Mazzarella, F., and Vespe, M. (2015). "Spatio-temporal data mining for maritime situational awareness." OCEANS 2015-Genova, IEEE, 1-8.

Arguedas, V. F., Pallotta, G., and Vespe, M. (2014). "Unsupervised maritime pattern analysis to enhance contextual awareness." Context-Awareness in Geographic Information Services (CAGIS 2014), 50.

Avram, V. R. (2012). "A spatio-temporal data representation framework with applications to anomaly detection in the maritime domain." Ph.D. thesis, Applied Science: School of Computing Science, Applied Science: School of Computing Science.

Balci, M. and Pegg, R. (2006). "Towards Global Maritime Domain Awareness-" Recent Developments and Challenges"." Information Fusion, 2006 9th International Conference on, IEEE, 1-5.

Bloisi, D. D., Iocchi, L., Nardi, D., and Fiorini, M. (2015). "Integrated visual information for maritime surveillance." Proc. Clean Mobility Intell. Transp. Syst., 237-264.

Bloisi, D. D., Previtali, F., Pennisi, A., Nardi, D., and Fiorini, M. (2017). "Enhancing automatic maritime surveillance systems with visual information." IEEE Transactions on Intelligent Transportation Systems, 18(4), 824-833.

Bomberger, N. A., Rhodes, B. J., Garagic, D., Dankert, J. R., Zandipour, M., Stolzar, L. H., Castan, G. D., and Seibert, M. (2008). "Adaptive spatial scale for cognitivelyinspired motion pattern learning \& analysis algorithms for higher-level fusion and automated scene understanding." Military Communications Conference, 2008. MILCOM 2008. IEEE, IEEE, 1-7.

Bomberger, N. A., Rhodes, B. J., Seibert, M., and Waxman, A. M. (2006). "Associative learning of vessel motion patterns for maritime situation awareness." Information Fusion, 2006 9th International Conference on, IEEE, 1-8.

Boraz, S. C. (2009). "Maritime domain awareness: Myths and realities." Naval War College Review, 62(3), 137.

Braca, P., Grasso, G., Vespe, M., Maresca, S., and Horstmann, J. (2012). "Application of the JPDA-UKF to HFSW radars for maritime situational awareness." 15th International Conference on Information Fusion (FUSION), 2585-2592.

Braca, P., Maresca, S., Grasso, R., Bryan, K., and Horstmann, J. (2015). "Maritime surveillance with multiple over-the-horizon HFSW radars: An overview of recent experimentation." IEEE Aerospace and Electronic Systems Magazine, 30(12), 4-18.

Brax, C. (2011). "Anomaly detection in the surveillance domain." Ph.D. thesis, rebro 
universitet, rebro universitet.

Brdje, A. (2012). "Hello, is there anybody out therejust nod if you can hear me.

Buck, H., Sharghi, E., Guilas, C., Stastny, J., Morgart, W., Schalcosky, B., and Pifko, K. (2008). "Enhanced ship detection from overhead imagery." Optics and Photonics in Global Homeland Security IV, Vol. 6945, International Society for Optics and Photonics, 69450U.

Burrell, J. (2016). "How the machine thinks: Understanding opacity in machine learning algorithms." Big Data \& Society, 3(1), 2053951715622512.

Carthel, C., Coraluppi, S., Grasso, R., and Grignan, P. (2007). "Fusion of AIS, RADAR and SAR data for maritime surveillance." Proceedings of SPIE - The International Society for Optical Engineering, Vol. 6748, Society of Photo-Optical Instrumentation Engineers, 67480Y-1.

Caschili, S., Medda, F., Parola, F., and Ferrari, C. (2014). "An analysis of shipping agreements: The cooperative container network." Networks and Spatial Economics, 14(3), 357-377.

Cazzanti, L. and Pallotta, G. (2015). "Mining maritime vessel traffic: Promises, challenges, techniques." OCEANS 2015-Genova, IEEE, 1-6.

Chandola, V., Banerjee, A., and Kumar, V. (2009). "Anomaly detection: A survey." ACM computing surveys (CSUR), 41(3), 15.

Colmanta, A. and van Vuurena, J. H. "Design of a Decision Support System for Maritime Law Enforcement Response Selection, system.

COM-EU (2009). "Towards the integration of maritime surveillance: A common information sharing environment for the eu maritime domain (10). 538 final.

C.Zor, J. (2017). "Maritime anomaly detection in ferry tracks." ICASSP, IEEE International Conference on Acoustics, Speech and Signal Processing - Proceedings.

Dai, Y. M. and Liu, K. S. (2015). "TACSA: A web-service based system for coastal surveillance and situational awareness." Security Technology (ICCST), 2015 International Carnahan Conference on, IEEE, 409-413.

Davenport, M. (2009). "Literature and product review of visual analytics for maritime awareness." Report no., MACDONALD DETTWILER AND ASSOCIATES LTD RICHMOND (CANADA).

de Vries, G. and van Someren, M. (2013). "Recognizing Vessel Movements from Historical Data." Situation Awareness with Systems of Systems, Springer, 105-118.

Dimitrova, T., Mazzola, L., and Tsois, A. (2013a). "An Interactive Web-Based Geographical System in Assisting Location Matching Decisions.

Dimitrova, T., Tsois, A., and Camossi, E. (2014). "Development of a Web-Based Geographical Information System for Interactive Visualization and Analysis of Container Itineraries." Development, 3(01).

Dimitrova, T. V., Tsois, A., and Camossi, E. (2013b). "Visualization of container movements through a web-based geographical information system." Intelligence and Security Informatics Conference (EISIC), 2013 European, IEEE, 182-185.

Dobrkovic, A., Iacob, M.-E., and van Hillegersberg, J. (2018). "Maritime pattern extraction and route reconstruction from incomplete ais data." International Journal of Data Science and Analytics.

Dragos, V. (2016). "From Finding to Explaining: Information Retrieval to Support Maritime Anomaly Analysis." International Journal of Knowledge and Systems Science (IJKSS), 7(1), 40-55. 
Pre-peer reviewed version.

Riveiro M., Pallotta G., and Vespe M. Maritime anomaly detection: A review. WIREs Data Mining Knowl Discov.

Advanced Review. 2018;e1266.

Edlund, J., Gronkvist, M., Lingvall, A., and Sviestins, E. (2006). "Rule-based situation assessment for sea surveillance." Proceedings of the SPIE-The International Society for Optical Engineering, USA, Vol. 6242, 624203-1.

Ekman, J. and Holst, A. (2008). "Incremental stream clustering and anomaly detection.

Etienne, L., Ray, C., and McARDLE, G. (2011). "Spatio-Temporal Visualisation of Outliers." Proceedings of the international workshop on Maritime Anomaly Detection (MAD), 119.

Fooladvandi, F., Brax, C., Gustavsson, P., and Fredin, M. (2009). "Signature-based activity detection based on Bayesian networks acquired from expert knowledge." Information Fusion, 2009. FUSION'09. 12th International Conference on, IEEE, 436-443.

Fridman, N., Amir, D., Schvartzman, I., Kleinerman, I., Sulkovski, Y., Gorlik, Z., and Kligsberg, S. (2017). "KINGFISHER: Total Maritime Awareness System, system." Proceedings of the 16th Conference on Autonomous Agents and MultiAgent Systems, International Foundation for Autonomous Agents and Multiagent Systems, 17841786.

Galdorisi, G. and Goshorn, R. (2005). "Bridging the policy and technology gap: A process to instantiate maritime domain awareness." OCEANS, 2005. Proceedings of MTS/IEEE, IEEE, 1-8.

Garnier, B. and Napoli, A. (2016). "Exploiting the Potential of the Future Maritime Big Data." Maritime Knowledge Discovery and Anomaly Detection Workshop, 24-27.

Graham, R. (2009). "MDA Challenges for Operational Research and Analysis." Report no., DEFENCE RESEARCH AND DEVELOPMENT CANADA OTTAWA (ONTARIO).

Griffiths, H. and Baker, C. (2005). "Passive coherent location radar systems. part 1: Performance prediction." IEE Proceedings-Radar, Sonar and Navigation, 152, 153159.

Hanna, M. J. (2015). "Encounter detection using visual analytics to improve maritime domain awareness." Report no., NAVAL POSTGRADUATE SCHOOL MONTEREY CA.

Hayes, M. A. and Capretz, M. A. (2015). "Contextual anomaly detection framework for big sensor data." Journal of Big Data, 2(1), 2.

Helldin, T. and Riveiro, M. (2009). "Explanation Methods for Bayesian Networks: review and application to a maritime scenario." Proceedings of the 3rd Annual Skvde Workshop on Information Fusion Topics (SWIFT 2009), 11-16.

Hiemstra, H. (2008). "Defining a Balanced Maritime Safety and Security System." INCOSE International Symposium, Vol. 18, Wiley Online Library, 2088-2101.

Horn, S., Eisler, C., Dobias, P., and Collins, N. J. (2016). "Data requirements for anomaly detection." Maritime Knowledge Discovery and Anomaly Detection Workshop Proceedings, Publications Office of the European Union, 52-56.

Horstmann, J., Grasso, R., Coffin, M., Gurgel, K. W., and Schlick, T. (2010). "Evaluation of an HF-radar Ship Detection Algorithm by Comparison to AIS and SAR data." Proceedings of IEEE IGARSS Conference, Honolulu, Hawaii.

Hunter, A. (2009). "Belief modeling for maritime surveillance." 2009 12th International Conference on Information Fusion, FUSION 2009, 1926-1932.

Jaszewski, M., Parameswaran, S., Hallenborg, E., and Bagnall, B. (2015). "Evaluation of maritime object detection methods for full motion video applications using 
Pre-peer reviewed version.

Riveiro M., Pallotta G., and Vespe M. Maritime anomaly detection: A review. WIREs Data Mining Knowl Discov.

Advanced Review. 2018;e1266.

the PASCAL VOC Challenge framework, comparative." Proceedings of SPIE - The International Society for Optical Engineering, S. E. Loce R.P., ed., Vol. 9407, SPIE. Jousselme, A.-L. and Pallotta, G. (2015). "Dissecting uncertainty-based fusion techniques for maritime anomaly detection." 2015 18th International Conference on Information Fusion, Fusion 2015, Institute of Electrical and Electronics Engineers Inc., $34-41$.

Kanellopoulos, S. A., Katsoulis, S., Motos, D., Lampropoulos, V., Margonis, C., Dimitros, K., and Thomopoulos, S. C. (2015). "OCULUS Sea INTEGRATED MARITIME SURVEILLANCE PLATFORM, system." Proc. of SPIE Vol, Vol. 9474, $94740 \mathrm{~N}-1$.

Katsilieris, F., Braca, P., and Coraluppi, S. (2013a). "Detection of malicious AIS position spoofing by exploiting radar information." Information fusion (FUSION), 2013 16th international conference on, IEEE, 1196-1203.

Katsilieris, F., Braca, P., and Coraluppi, S. (2013b). "Detection of malicious ais position spoofing by exploiting radar information." Information fusion (FUSION), 2013 16th international conference on, IEEE, 1196-1203.

Kazemi, S., Abghari, S., Lavesson, N., Johnson, H., and Ryman, P. (2013). "Open data for anomaly detection in maritime surveillance." Expert Systems with Applications, 40(14), 5719-5729.

Keane, K. R. (2017). "Detecting motion anomalies." Proceedings of the 8th ACM SIGSPATIAL Workshop on GeoStreaming, IWGS'17, New York, NY, USA, ACM, 21-28.

Keim, D., Kohlhammer, J., Ellis, G., and Mansmann, F. (2010). "Mastering the Information Age: Solving Problems with Visual Analytics." Eurographics, Vol. 2, 5.

Khatkhate, A. M., Ray, A., and Keller, E. (2007). "Modelling and system identification of an experimental apparatus for anomaly detection in mechanical systems." Applied mathematical modelling, 31(4), 734-748.

Kowalska, K. and Peel, L. (2012). "Maritime anomaly detection using Gaussian Process active learning." 15th International Conference on Information Fusion, FUSION 2012, IEEE, 1164-1171.

Kraiman, J., Arouh, S., and Webb, M. (2002). "Automated anomaly detection processor." Proceedings of SPIE-The International Society for Optical Engineering, 4716, $128-137$.

Lane, R., Nevell, D., Hayward, S., and Beaney, T. (2010). "Maritime anomaly detection and threat assessment." 13th Conference on Information Fusion, Fusion 2010, IEEE, $1-8$.

Lane, R. O. (2011). "Maritime route anomaly detection." MAD 2011 Workshop Proceedings, 123.

Langellotti, D., Colone, F., Lombardo, P., Tilli, E., Sedehi, M., and Farina, A. (2014). "Over the horizon maritime surveillance capability of DVB-T based passive radar." 44th European Microwave Conference (EuMC), 1812-1815.

Lavigne, V. (2014a). "Interactive visualization applications for maritime anomaly detection and analysis." Defence Research Reports, Technical report.

Lavigne, V. (2014b). Maritime Visual Analytics Prototype. Defence Research and Development Canada.

Lavigne, V. and Gouin, D. (2011). "Applicability of visual analytics to defence and security operations." Report no., DEFENCE RESEARCH AND DEVELOPMENT 
Pre-peer reviewed version.

Riveiro M., Pallotta G., and Vespe M. Maritime anomaly detection: A review. WIREs Data Mining Knowl Discov.

Advanced Review. 2018;e1266.

CANADA VALCARTIER (QUEBEC).

Laxhammar, R. (2008). "Anomaly detection for sea surveillance." Information Fusion, 2008 11th International Conference on, IEEE, 1-8.

Laxhammar, R. (2011). "Anomaly detection in trajectory data for surveillance applications." Ph.D. thesis, rebro universitet, rebro universitet.

Laxhammar, R. and Falkman, G. (2010). "Conformal prediction for distributionindependent anomaly detection in streaming vessel data." Proceedings of the First International Workshop on Novel Data Stream Pattern Mining Techniques, ACM, $47-55$.

Laxhammar, R., Falkman, G., and Sviestins, E. (2009). "Anomaly detection in sea traffic-a comparison of the gaussian mixture model and the kernel density estimator." Information Fusion, 2009. FUSION'09. 12th International Conference on, IEEE, 756-763.

Le Guillarme, N. and Lerouvreur, X. (2013). "Unsupervised extraction of knowledge from S-AIS data for maritime situational awareness." Proceedings of the 16th International Conference on Information Fusion, FUSION 2013, 2025-2032.

Lee, B., Park, N., and Kim, J. (2014). "A Requirement Analysis of Awareness-Based Vessel Traffic Service System for Maritime Safety." Advances in Computer Science and its Applications, Springer, 379-384.

Lei, P.-R. (2016). "A framework for anomaly detection in maritime trajectory behavior." Knowledge and Information Systems, 47(1), 189-214.

Lei, P.-R., Tsai, T.-H., Wen, Y.-T., and Peng, W.-C. (2017). "ConflictFinder: Mining Maritime Traffic Conflict from Massive Ship Trajectories, system." Mobile Data Management (MDM), 2017 18th IEEE International Conference on, IEEE, 356-357.

Li, X., Han, J., Kim, S., and Gonzalez, H. (2007). "Roam: Rule-and motif-based anomaly detection in massive moving object data sets, framework." Proceedings of the 2007 SIAM International Conference on Data Mining, SIAM, 273-284.

Liu, B., De Souza, E., Hilliard, C., and Matwin, S. (2015). "Ship movement anomaly detection using specialized distance measures." 2015 18th International Conference on Information Fusion, Fusion 2015, Institute of Electrical and Electronics Engineers Inc., $1113-1120$.

MacLeod, M. R. and Wardrop, W. M. (2015). "Operational Analysis at Combined Maritime Forces.

Malhotra, B., Tan, W.-J., Cao, J., Kister, T., Bressan, S., and Tan, K.-L. (2011). "ASSIST: access controlled ship identification streams, system." Proceedings of the 19th ACM SIGSPATIAL International Conference on Advances in Geographic Information Systems, ACM, 485-488.

Malik, A., Maciejewski, R., Jang, Y., Oliveros, S., Yang, Y., Maule, B., White, M., and Ebert, D. S. (2014). "A visual analytics process for maritime response, resource allocation and risk assessment." Information Visualization, 13(2), 93-110.

Martineau, E. and Roy, J. (2011). "Maritime anomaly detection: Domain introduction and review of selected literature." Report no., DEFENCE RESEARCH AND DEVELOPMENT CANADA VALCARTIER (QUEBEC).

Mascaro, S., Korb, K. B., and Nicholson, A. E. (2010). "Learning abnormal vessel behaviour from ais data with bayesian networks at two time scales." Tracks a Journal of Artists Writings.

Mascaro, S., Nicholson, A., and Korb, K. (2014). "Anomaly detection in vessel tracks 
using Bayesian networks." International Journal of Approximate Reasoning, 55(1 PART 1), 84-98.

Mazzarella, F., Vespe, M., Alessandrini, A., Tarchi, D., Aulicino, G., and Vollero, A. (2017). "A novel anomaly detection approach to identify intentional AIS on-off switching." Expert Systems with Applications, 78, 110-123.

Mazzarella, F., Vespe, M., Damalas, D., and Osio, G. (2014). "Discovering vessel activities at sea using AIS data: Mapping of fishing footprints." 17th International Conference on information Fusion (FUSION).

Mazzarella, F., Vespe, M., Tarchi, D., Aulicino, G., and Vollero, A. (2016). "AIS reception characterisation for AIS on/off anomaly detection." FUSION 2016 - 19th International Conference on Information Fusion, Proceedings, Institute of Electrical and Electronics Engineers Inc., 1867-1873.

Moreira, R. D. S., Ebecken, N. F. F., Alves, A. S., Livernet, F., and Campillo-Navetti, A. (2014). "A survey on video detection and tracking of maritime vessels." International Journal of Research and Reviews in Applied Sciences, 20(1), 37.

Morel, M., Georg, J.-P., Littaye, A., Jangal, F., Napoli, A., Giraud, M.-A., and Lebrevelec, J. (2008). "ScanMaris: automatic detection of abnormal vessel behaviours, system." Colloque international 3AF:" L'Europe et la transformation de ses forces", 8 -pages.

Nandan, N., Malhotra, B., and Dahlmeier, D. (2013). "sRADAR: A complex event processing and visual analytics system for maritime intelligence." Conferences in Research and Practice in Information Technology Series, Y. Zhao, A. Stranieri, L. Liu, P. Kennedy, P. Christen, K.-L. Ong, and Y. Zhao, eds., Vol. 146, Australian Computer Society, 35-40.

Natale, F., Gibin, M., Alessandrini, A., Vespe, M., and Paulrud, A. (2015). "Mapping fishing effort through ais data." PloS one, 10.

Nilsson, M., Van Laere, J., Ziemke, T., and Edlund, J. (2008). "Extracting rules from expert operators to support situation awareness in maritime surveillance." Information Fusion, 2008 11th International Conference on, IEEE, 1-8.

Nordmo Skauen, A., Helleren, O., Olsen, O., and Olsen, R. (2013). "Operator and User Perspective of Fractionated AIS Satellite Systems.

Nuutinen, M., Savioja, P., and Sonninen, S. (2007). "Challenges of developing the complex socio-technical system: Realising the present, acknowledging the past, and envisaging the future of vessel traffic services." Applied ergonomics, 38(5), 513-524.

Olson, D. R. (2006). "Maritime Domain Awareness.." Report no., Sandia National Laboratories (SNL-NM), Albuquerque, NM (United States).

Osekowska, E. and Carlsson, B. (2009). "Visualizing anomalies and traffic rules in a maritime setting using potential fields." DESIGN AND IMPLEMENTATION OF A MARITIME TRAFFIC MODELING AND ANOMALY DETECTION METHOD, 95.

Osekowska, E. and Carlsson, B. (2015). "Learning maritime traffic rules using potential fields." Lecture Notes in Computer Science (including subseries Lecture Notes in Artificial Intelligence and Lecture Notes in Bioinformatics), 9335, 298-312.

Pallotta, G., Horn, S., Braca, P., and Bryan, K. (2014). "Context-enhanced vessel prediction based on Ornstein-Uhlenbeck processes using historical AIS traffic patterns: Real-world experimental results, context." FUSION 2014 - 17th International Conference on Information Fusion, Institute of Electrical and Electronics Engineers Inc., 
$1-7$.

Pallotta, G. and Jousselme, A.-L. (2015). "Data-driven detection and context-based classification of maritime anomalies." 2015 18th International Conference on Information Fusion, Fusion 2015, Institute of Electrical and Electronics Engineers Inc., 1152-1159.

Pallotta, G., Vespe, M., and Bryan, K. (2013a). "Traffic knowledge discovery from AIS data." Proceedings of the 16th International Conference on Information Fusion, FUSION 2013, 1996-2003.

Pallotta, G., Vespe, M., and Bryan, K. (2013b). "Traffic route extraction and anomaly detection from ais data." International COST MOVE Workshop on Moving Objects at Sea.

Pallotta, G., Vespe, M., and Bryan, K. (2013c). "Traffic route extraction and anomaly detection (TREAD): Vessel pattern knowledge discovery and exploitation for maritime situational awareness." NATO Formal Report CMRE-FR-2013-001, NATO Unclassified.

Pallotta, G., Vespe, M., and Bryan, K. (2013d). "Vessel pattern knowledge discovery from AIS data: A framework for anomaly detection and route prediction." Entropy, 15(6), 2218-2245.

Papi, F., Tarchi, D., Vespe, M., Oliveri, F., Borghese, F., Aulicino, G., and Vollero, A. (2014). "Radiolocation and tracking of automatic identification system signals for maritime situational awareness." IET Radar, Sonar \& Navigation, 9(5), 568-580.

Patroumpas, K., Alevizos, E., Artikis, A., Vodas, M., Pelekis, N., and Theodoridis, Y. (2017). "Online event recognition from moving vessel trajectories." GeoInformatica, 21(2), 389-427.

Portnoy, L., Eskin, E., and Stolfo, S. (2001). "Intrusion detection with unlabeled data using clustering." In Proceedings of ACM CSS Workshop on Data Mining Applied to Security (DMSA-2001, Citeseer.

Radon, A., Wang, K., Glasser, U., Wehn, H., and Westwell-Roper, A. (2015). "Contextual verification for false alarm reduction in maritime anomaly detection, context." Proceedings - 2015 IEEE International Conference on Big Data, IEEE Big Data 2015, K. Luo, F.and Ogan, M. Zaki, L. Haas, B. Ooi, V. Kumar, S. Rachuri, S. Pyne, H. Ho, X. Hu, M.-I. Yu, S.and Hsiao, and J. Li, eds., Institute of Electrical and Electronics Engineers Inc., 1123-1133.

Radon, A. N. (2015). "False alarm reduction in maritime anomaly detection with contextual verification." Ph.D. thesis, Applied Sciences: School of Computing Science, Applied Sciences: School of Computing Science.

Ray, C., Gallen, R., Iphar, C., Napoli, A., and Bouju, A. (2015). "DeAIS project: Detection of AIS spoofing and Resulting Risks, system." OCEANS 2015-Genova, IEEE, 1-6.

Rhodes, B., Bomberger, N., Zandipour, M., Waxman, A., and Seibert, M. (2007a). "Cognitively-inspired motion pattern learning \& analysis algorithms for higher-level fusion and automated scene understanding." Proceedings - IEEE Military Communications Conference MILCOM, IEEE, 1-6.

Rhodes, B. J., Bomberger, N. A., Freyman, T. M., Kreamer, W., Kirschner, L., LItalien, A. C., Mungovan, W., Stauffer, C., Stolzar, L., Waxman, A. M., and others (2007b). "SeeCoast: persistent surveillance and automated scene understanding for ports and coastal areas, system." Proceedings of SPIE, Vol. 6578, 65781M. 
Rhodes, B. J., Bomberger, N. A., Seibert, M., and Waxman, A. M. (2005). "Maritime situation monitoring and awareness using learning mechanisms." Military Communications Conference, 2005. MILCOM 2005. IEEE, IEEE, 646-652.

Rhodes, B. J., Bomberger, N. A., Seibert, M., and Waxman, A. M. (2006). "SeeCoast: Automated port scene understanding facilitated by normalcy learning, system." Military Communications Conference, 2006. MILCOM 2006. IEEE, IEEE, 1-7.

Rhodes, B. J., Bomberger, N. A., and Zandipour, M. (2007c). "Probabilistic associative learning of vessel motion patterns at multiple spatial scales for maritime situation awareness." Information Fusion, 2007 10th International Conference on, IEEE, 1-8.

Ribeiro, M. T., Singh, S., and Guestrin, C. (2016). "Why should i trust you?: Explaining the predictions of any classifier." Proc. of the 22nd SIGKDD Int. Conf. on Knowledge Discovery and Data Mining, ACM, 1135-1144.

Ristic, B. (2014). "Detecting anomalies from a multitarget tracking output." IEEE Transactions on Aerospace and Electronic Systems, 50(1), 798-803.

Ristic, B., La Scala, B., Morelande, M., and Gordon, N. (2008). "Statistical analysis of motion patterns in AIS data: Anomaly detection and motion prediction." Proceedings of the 11th International Conference on Information Fusion, FUSION 2008, IEEE, $1-7$.

Riveiro, M. (2013). "The importance of visualization and interaction in the anomaly detection process." Innovative Approaches of Data Visualization and Visual Analytics, 133.

Riveiro, M. (2014). "Evaluation of normal model visualization for anomaly detection in maritime traffic." ACM Transactions on Interactive Intelligent Systems, 4(1), 5.

Riveiro, M. and Falkman, G. (2009). "Interactive visualization of normal behavioral models and expert rules for maritime anomaly detection." Proceedings of the 2009 6th International Conference on Computer Graphics, Imaging and Visualization: New Advances and Trends, CGIV2009, IEEE, 459-466.

Riveiro, M. and Falkman, G. (2010). "Supporting the analytical reasoning process in maritime anomaly detection: Evaluation and experimental design." Proceedings of the International Conference on Information Visualisation, IEEE, 170-178.

Riveiro, M., Falkman, G., and Ziemke, T. (2008). "Improving maritime anomaly detection and situation awareness through interactive visualization." Proceedings of the 11th International Conference on Information Fusion, FUSION 2008, IEEE, 1-8.

Riveiro, M., Falkman, G., Ziemke, T., and Kronhamn, T. (2009a). "Reasoning about anomalies: A study of the analytical process of detecting and identifying anomalous behavior in maritime traffic data." Proceedings of SPIE - The International Society for Optical Engineering, Vol. 7346, SPIE.

Riveiro, M., Falkman, G., Ziemke, T., and Warston, H. (2009b). "VISAD: An interactive and visual analytical tool for the detection of behavioral anomalies in maritime traffic data, system." Proceedings of SPIE - The International Society for Optical Engineering, Vol. 7346.

Riveiro, M. J. (2011). "Visual analytics for maritime anomaly detection." Ph.D. thesis, Örebro universitet, Örebro universitet.

Roberts, S. (2014). "Anomaly detection in vessel track data." Ph.D. thesis, University of Oxford, University of Oxford.

Rossi, A., Riccobono, A., and Landini, S. (2014). "Sun-glint false alarm mitigation in a maritime scenario." Proceedings of SPIE - The International Society for Optical 
Engineering, G. Bishop, G. Kamerman, J. Gonglewski, A. Killey, and O. Steinvall, eds., Vol. 9250, SPIE, 92500X-92500X.

Rothkrantz, L. and Scholte, K. (2013). "A surveillance system of a military harbour using an Automatic Identification System." Proceedings of the 14th International Conference on Computer Systems and Technologies, ACM, 169-176.

Roy, J. (2008). "Anomaly detection in the maritime domain." Proceedings of SPIE The International Society for Optical Engineering, Vol. 6945.

Roy, J. (2010). "Rule-based expert system for maritime anomaly detection." Proceedings of SPIE - The International Society for Optical Engineering, Vol. 7666, 76662N$76662 \mathrm{~N}$.

Roy, J. and Davenport, M. (2009). "Categorization of maritime anomalies for notification and alerting purpose." NATO workshop on data fusion and anomaly detection for maritime situational awareness, La Spezia, Italy, 15-17.

Roy, J. and Davenport, M. (2010). "Exploitation of maritime domain ontologies for anomaly detection and threat analysis." 2010 International Waterside Security Conference, WSS 2010, IEEE, 1-8.

Rudin, C. (2014). "Algorithms for interpretable machine learning." Proc. of the 20th SIGKDD Int. Conf. on Knowledge Discovery and Data Mining, ACM, 1519-1519.

Sacha, D., Sedlmair, M., Zhang, L., Lee, J. A., Weiskopf, D., North, S., and Keim, D. (2016). "Human-centered machine learning through interactive visualization." Proc. of European Symposium on Artificial Neural Networks (ESANN), Computational Intelligence and Machine Learning, 641-646.

Sermi, F., Mugnai, C., Cuccoli, F., and Facheris, L. (2013). "Analysis of the radar coverage provided by a maritime Radar Network of Co-operative Vessels based on real AIS data." Radar Conference (EuRAD), 2013 European, IEEE, 251-254.

Shafer, S., Harguess, J., and Forero, P. A. (2015). "Sparsity-driven anomaly detection for ship detection and tracking in maritime video." Automatic Target Recognition $X X V$, Vol. 9476, International Society for Optics and Photonics, 947608.

Shahir, H., Glasser, U., Nalbandyan, N., and Wehn, H. (2014). "Maritime situation analysis: A multi-vessel interaction and anomaly detection framework, system." Proceedings - 2014 IEEE Joint Intelligence and Security Informatics Conference, JISIC 2014, Institute of Electrical and Electronics Engineers Inc., 192-199.

Sidibé, A. and Shu, G. (2017). "Study of automatic anomalous behaviour detection techniques for maritime vessels." The Journal of Navigation, 70(4), 847-858.

Singh, A. K., Aggarwal, V., and Tiwari, P. (2016). "Coastal surveillance in multi sensor environment: A design approach, system." Recent Advances in Information Technology (RAIT), 2016 3rd International Conference on, IEEE, 1-6.

Snidaro, L., Garca, J., and Llinas, J. (2015a). "Context-based Information Fusion: A survey and discussion, context." Information Fusion, 25, 16 - 31.

Snidaro, L., Visentini, I., and Bryan, K. (2015b). "Fusing uncertain knowledge and evidence for maritime situational awareness via Markov Logic Networks." Information Fusion, 21(1), 159-172.

St-Hilaire, M.-O. and Hadzagic, M. (2013). "Information Mining Technologies to Enable Discovery of Actionable Intelligence to Facilitate Maritime Situational Awareness: IMINE, system." Report no., OODA Technologies Inc Montreal, QC Canada.

Stryna, M., Ma \lyszko, J., Wcel, K., Filipiak, D., and Abramowicz, W. (2016). "Architecture of maritime awareness system supplied with external information." Annual 
of Navigation, 23(1), 135-149.

Thomas, J. and Cook, K. (2005). Illuminating the Path: The Research and Development Agenda for Visual Analytics. IEEE Computer Society Press.

Tu, E., Zhang, G., Rachmawati, L., Rajabally, E., and Huang, G.-B. (2016). "Exploiting AIS data for intelligent maritime navigation: A comprehensive survey." arXiv preprint arXiv:1606.00981.

Vandecasteele, A., Devillers, R., and Napoli, A. (2013). "A semi-supervised learning framework based on spatio-temporal semantic events for maritime anomaly detection and behavior analysis." CoastGIS 2013-The 11th International Symposium for GIS and Computer Cartography for Coastal Zone Management, 4-pages.

Vandecasteele, A. and Napoli, A. (2012a). "Enhancement of ontology with spatial reasoning capabilities to support maritime anomaly detection." System of Systems Engineering (SoSE)., Genoa, Italie.

Vandecasteele, A. and Napoli, A. (2012b). "Spatial ontologies for detecting abnormal maritime behaviour." Program Book - OCEANS 2012 MTS/IEEE Yeosu: The Living Ocean and Coast - Diversity of Resources and Sustainable Activities, IEEE, 1-7.

Varfis, A., Kotsakis, E., Tsois, A., Donati, A., Sjachin, M., Camossi, E., Villa, P., Dimitrova, T., and Pellissier, M. (2011). "ConTraffic: Maritime Container Traffic Anomaly Detection, proceedings of the First International Workshop on Maritime Anomaly Detection (MAD), system." June, 17, 13-14.

Varga, M. and Lavigne, V. (2016). "Application of Visual Analytics to Maritime Domain Analysis.

Vatin, G. and Napoli, A. (2013a). "Guiding the controller in geovisual analytics to improve maritime surveillance." GEOProcessing 2013: the Fifth International Conference on Advanced Geographic Information Systems, Applications, and Services, IARIA, 26-31.

Vatin, G. and Napoli, A. (2013b). "High-Level Taxonomy of Geovisual Analytics Tasks for Maritime Surveillance." ICC 2013 - 26th International Cartographic Conference, Dresde, Germany, 14 pages (August).

Vatin, G. and Napoli, A. (2013c). "Toward user-centred geovisual analytics in maritime surveillance." GeoViz 2013: Geovisualization and Cognitive Visualization the GeoViz_Hamburg Workshop, 2-pages.

Vespe, M. and Greidanus, H. (2012). "Sar image quality assessment and indicators for vessel and oil spill detection." IEEE Transactions on Geoscience and Remote Sensing, 50(11), 4726-4734.

Vespe, M., Greidanus, H., and Alvarez, M. (2015). "The declining impact of piracy on maritime transport in the indian ocean: Statistical analysis of 5-year vessel tracking data." Marine Policy, 9-15.

Vespe, M., Pallotta, G., Visentini, I., Bryan, K., and Braca, P. (2012). "Maritime anomaly detection based on historical trajectory mining." Proceedings of the NATO Port and Regional Maritime Security Symposium, 1-11.

Vespe, M., Sciotti, M., and Battistello, G. (2008). "Multi-sensor autonomous tracking for maritime surveillance." Radar, 2008 International Conference on, IEEE, 525-530.

Wan, Z., Chen, J., Makhloufi, A. E., Sperling, D., and Chen, Y. (2016). "Four routes to better maritime governance." Nature News, 540(7631), 27.

Wang, G., Malik, A., Yau, C., Surakitbanharn, C., and Ebert, D. S. (2017). "TraSeer: A visual analytics tool for vessel movements in the coastal areas, system." Technologies 
for Homeland Security (HST), 2017 IEEE International Symposium on, IEEE, 1-6. Wang, X., Liu, X., Liu, B., De Souza, E., and Matwin, S. (2014). "Vessel route anomaly detection with Hadoop MapReduce." Proceedings - 2014 IEEE International Conference on Big Data, IEEE Big Data 2014, W. Chang, J. Huan, N. Cercone, S. Pyne, J. Honavar, V.and Lin, X. Hu, C. Aggarwal, B. Mobasher, J. Pei, and N. R., eds., Institute of Electrical and Electronics Engineers Inc., 25-30.

Wen, Y., Lai, C., Lei, P., and Peng, W. (2014). "RouteMiner: Mining ship routes from a massive maritime trajectories, system." Proceedings - IEEE International Conference on Mobile Data Management, Vol. 1, Institute of Electrical and Electronics Engineers Inc., 353-356.

White, B. E. and Semy, S. K. (2010). "Case study: Maritime domain awareness." Systems Conference, 2010 4th Annual IEEE, IEEE, 575-580.

Wiersma, J. (2010). "Assessing vessel traffic service operator situation awareness." Doctoral dissertation, Safety Science. Technology, Policy and Management, Boxpress, Oisterwijk.

Willems, N., Scheepens, R., van de Wetering, H., and van Wijk, J. J. (2013). "Visualization of vessel traffic." Situation Awareness with Systems of Systems, Springer, 73-87.

Willems, N., Van De Wetering, H., and Van Wijk, J. J. (2009). "Visualization of vessel movements." Computer Graphics Forum, Vol. 28, Wiley Online Library, 959-966.

Willems, N., van Hage, W. R., de Vries, G., Janssens, J. H., and Malais, V. (2010). "An integrated approach for visual analysis of a multisource moving objects knowledge base." International Journal of Geographical Information Science, 24(10), 1543-1558.

Zhou, J., Li, Z., Wang, Y., and Chen, F. (2013). "Transparent machine learningrevealing internal states of machine learning." Proc. of IUI2013 Workshop on Interactive Machine Learning, 1-3.

Zissis, D., Xidias, E., and Lekkas, D. (2015). "A cloud based architecture capable of perceiving and predicting multiple vessel behaviour, system." Applied Soft Computing Journal, 35, 652-661.

\section{APPENDIX}

In the following appendix, details on the search, selection process and working steps are given. Google Scholar, IEEE Xplore, Scopus and ScienceDirect were used during the literature search. The main keywords used were maritime anomaly detection, and the search was restricted between 1996-2017; the software used was Zotero and JabRef. The steps, keywords and search strings are as follows.

\section{First step}

- Google Scholar. Export to Zotero after title and abstract. 98 pages (10 entries each): 980 of which 479 were selected (based on title and abstract reading).

- IEEE Xplore: ((("Document Title": maritime) OR "Abstract": maritime) AND "Abstract" :anomaly detection), 1996-2017. Conference Publications (40), Journals and Magazines (1), Early Access Articles (1). 
- Scopus: TITLE-ABS-KEY ( maritime AND anomaly AND detection ) AND ( EXCLUDE ( LANGUAGE , "Chinese" ) OR EXCLUDE ( LANGUAGE , "French") ) AND ( LIMIT-TO ( SUBJAREA , "COMP" ) OR LIMIT-TO ( SUBJAREA , "ENGI") OR LIMIT-TO ( SUBJAREA, "MATH" ) OR LIMIT-TO ( SUBJAREA , "PHYS" ) OR LIMIT-TO ( SUBJAREA, "SOCI" ) OR LIMIT-TO ( SUBJAREA , "DECI") OR LIMIT-TO ( SUBJAREA , "ENVI" )) AND ( EXCLUDE ( SUBJAREA , "CENG") OR EXCLUDE ( SUBJAREA , "EART" ) ) Result Scopus: 135 Conference Paper (97), Article (19), Conference Review (15), Book (1), Book Chapter (1), Article in Press (1), Review (1). Total 135. Total library after Scopus: 658 .

- ScienceDirect: 37 total results of which 8 were selected Search results: 37 results found for pub-date i 1996 and (maritime anomaly detection) AND LIMIT-TO(cids, "271489, 271506, 314898, 271824, 271447, 272144, 271494, 271816, 271434, 272413, 271462, 271471, 280203, 271430, 271599, 271515, 271488","NDT AND E International,Expert Systems with Applications,IFAC Proceedings Volumes, Marine Policy, Acta Astronautica, Information Fusion, Journal of Loss Prevention in the Process Indus...,Ocean cite AND Coastal Management,Building and Environment, Mechanical Systems and Signal Processing, Ocean Engineering, Optics and Lasers in Engineering, Procedia Computer Science, Reliability Engineering AND System Safety, Robotics and Autonomous Systems, Computer Communications, Optics AND Laser Technology") AND LIMIT-TO (topics, "unite state, control, session chair, univ, safety, tech, vessel, ship, system, fusion, operator, production, risk, situation, abnormal, alaska, anomaly").

Total Maritime AD library: 667 items. Format .bib.

Second step. 255 entries identified by Zotero as duplicates were removed. After merging the duplicates, 522 were left.

Third step. Removed those entries that corresponded to introductory descriptions to conferences and workshops, irrelevant updates of reports, citation entires and other languages than English. Number of items after this step: 496.

Fourth step. Inspection of all full-text. Several entries were removed due to failing to find full-texts (internal reports), patents with too little information to describe the work (7), outside the scope, duplicates (very similar works published in several venues, or published both as conference, chapter or journal). Number of items after this step: 467. 